\title{
Calpain and AR-V7: Two potential therapeutic targets to overcome acquired docetaxel resistance in castration-resistant prostate cancer cells
}

\author{
LEI LIU ${ }^{1}$, NING LOU ${ }^{1}$, XIANG LI $^{1}$, GUANGHUA XU ${ }^{1}$, HAILONG RUAN $^{1}$, WEN XIAO ${ }^{1}$, BIN QIU ${ }^{1}$, \\ LIN BAO $^{1}$, CHANGFEI YUAN ${ }^{1}$, XINMIAN HUANG $^{1}$, KESHAN WANG $^{1}$, QI CAO $^{1}$, \\ KE CHEN $^{1}$, HONGMEI YANG ${ }^{2}$ and XIAOPING ZHANG ${ }^{1}$ \\ ${ }^{1}$ Department of Urology, Union Hospital, Tongji Medical College, Huazhong University of Science and Technology, \\ Wuhan 430022, China; ${ }^{2}$ Department of Pathogenic Biology, School of Basic Medicine, \\ Huazhong University of Science and Technology, Wuhan, Hubei 430030, P.R. China
}

Received October 7, 2016; Accepted January 26, 2017

DOI: 10.3892/or.2017.5623

\begin{abstract}
Docetaxel-based chemotherapy has been widely used as the first-line treatment for castration-resistant prostate cancer (CRPC) patients. However, the mechanisms of docetaxel-resistance remain unclear. In the present study with the establishment of 2 in vitro models of docetaxel-resistant CRPC cell sublines, we firstly reported that activation of calpain may play a promotional role in the resistance of docetaxel in prostate cancer, meanwhile using the calpain inhibitor combined with docetaxel improved the efficiency of docetaxel in docetaxel-resistant cell sublines. Moreover, we also found that the expression of androgen-independent constitutively and transcriptionally active androgen receptor splice variant-7 (AR-V7) remained high in the docetaxel-resistant CRPC cell subline Rvl-DR, and that it may be involved in acquired docetaxel-resistance of CRPC. However, a novel importin- $\beta$ inhibitor (importazole) was only capable of slightly decreasing the transcriptional activity of the AR signaling pathway via blocking nuclear import of AR-FL and various non-specific AR-Vs, instead of AR-V7. These findings suggest that calpain and AR-V7 may serve as important biomarkers in the treatment of CRPC, and targeting calpain and AR-V7 may provide a new approach in overcoming docetaxel-resistance.
\end{abstract}

\section{Introduction}

Prostate cancer is the second leading cause of cancer mortality in men in the US. Androgen deprivation therapy (ADT)

Correspondence to: Professor Xiaoping Zhang, Department of Urology, Union Hospital, Tongji Medical College, Huazhong University of Science and Technology, 1277 JieFang Avenue, Wuhan 430022, China

E-mail: xzhang@hust.edu.cn

Key words: prostate cancer, docetaxel, chemoresistance, calpain, AR-V7 which aims at decreasing androgen levels and/or blocking androgen binding with an androgen receptor (AR) remains the first-line treatment of advanced prostate cancer (1). Although, this approach works out satisfactorily in the early use, prostate cancer may eventually become uncontrollable, termed castration-resistant prostate cancer (CRPC). Since 2004, docetaxel-based chemotherapy has been established as the first-line therapy in patients with CRPC (2). Unfortunately, nearly half of the patients are initially docetaxel-insensitive, and the patients that are initially sensitive to docetaxel may acquire resistance to docetaxel within 1 year (3). In recent years, a number of new drugs have obtained FDA approval and have been used as the second-line therapy in CRPC, such as cabazitaxel, enzalutamide and abiraterone. However, after second-line treatment, resistance may still occur inevitably and the overall survival is only prolonged for $<5$ months (4). Therefore, a deeper understanding of the mechanisms of acquired docetaxel-resistance is extremely valuable.

Docetaxel belongs to taxane chemotherapeutic drugs. It can inhibit the mobility of microtubules via binding microtubules and preventing their depolymerization, consequently causing stagnation of mitosis and cell apoptosis (5). This mechanism was once thought to be the main mechanism of taxanes treatment for prostate cancer, until recently some research teams proved that taxanes also suppressed the AR signaling pathways. Taxanes block AR nuclear translocation, thus, inhibiting the expression of AR regulation genes (6). The discovery of this new mechanism indicates that taxanes and ADT drugs may have a synergistic effect, since both therapies inhibit the AR signaling pathway by targeting different compositions of the AR axis, for example, while abiraterone inhibits the generation of androgen, docetaxel can block nuclear import of AR. However, a new clinical study reported that the pharmacological activity of docetaxel was lower than expected in the patients who were under abiraterone application previously. Meanwhile, those who were under abiraterone application and acquired resistance did not respond to docetaxel treatment (7). This result suggests that the two classes of drugs in addition 
to sharing a common pharmacological mechanism (acting on the AR signaling pathway), may also share common resistance mechanisms. Recently, various laboratory studies and clinical studies have also suggested that there is a cross-resistance phenomenon between docetaxel and second-line ADT drugs (7-11).

Calpain is comprised of a family of relatively conservative intracellular cysteine proteases. Currently, a total of 14 calpain family members have been identified. Most of calpain activity is due to $\mu$-calpain and m-calpain, which are heterodimers comprised of a large $80 \mathrm{kDa}$ catalytic subunit (CAPN1 and CAPN2, respectively) and a common $28 \mathrm{kDa}$ regulatory subunit (CAPNS1) (12). In cancer cells, the expression of calpain is usually increased, since it can be used for cleaving the cytoskeleton in order to promote migration and invasion. In schwannoma and meningeoma, $\mu$-calpain is overexpressed (13). In clear cell renal cell carcinoma, the expression of CAPN1 mRNA is increased (14). In colorectal cancer, increased expression of $\mathrm{m}$-calpain has also been observed (15). However, discordant results have been presented for prostate cancer $(16,17)$. Recently, Liu et al indicated that long-term ADT induction in hormone-naïve prostate cancer (HNPC) cell line LNCaP had the ability to develop it into a more invasive CRPC cell subline, and calpain in this CRPC cell subline was hyperactivated. In addition, using calpeptin (a calpain inhibitor) suppressed the migration and invasion of this subline (18), reminding us that calpain plays an important role in the process of HNPC developing into CRPC. In another study, Jorfi et al demonstrated that when calpeptin was used in combination with docetaxel in prostate cancer cells, calpeptin increased the concentration of docetaxel by means of decreasing microvesicle release (19). Since calpain promotes ADT resistance and there is generally a cross-resistance phenomenon between docetaxel and ADT drugs, we explored whether calpain also plays a promoting role in acquired docetaxel-resistance.

Androgen receptor splicing variants (AR-Vs) which have been widely discussed lately, lack the ligand-binding domain (LBD). Furthermore, they can perform constitutive transcriptional activity in the absence of androgen and drive prostate cancer development in the direction of castration-resistance. In addition, these AR-Vs are generally overexpressed in CRPC compared with HNPC (20,21). AR-V7 (also known as AR3) is one of the major AR-Vs. CRPC patients with a high expression of AR-V7 have a relatively shorter overall survival (22). Other recent studies strongly confirmed that AR-V7 played an important role in the resistance of abiraterone and enzalutamide $(23,24)$. Although, the promoting role of AR-V7 concerning ADT-treatment resistance has been widely recognized, whether AR-V7 also plays a crucial role in the resistance of docetaxel remains unclear.

After 2 in vitro docetaxel-resistant prostate cancer cell sublines were successfully established, we focused on the resistance mechanisms of docetaxel in respect to calpain and AR-V7. Subsequently we studied the biological functions by targeting calpain and the influence on the transcriptional activity of the AR signaling pathway by targeting importin- $\beta$, in order to find new biomarkers for CRPC treatment from a new perspective as well as appropriate methods to overcome docetaxel-resistance in CRPC.

\section{Materials and methods}

Cell lines and chemicals. Human CRPC cell lines, PC3 and Rv1, human HNPC cell line, LNCaP, human benign prostatic hyperplasia cell line, BPH-1, were obtained from the American Type Culture Collection (ATCC; Manassas, VA, USA) and maintained in RPMI-1640 medium (Gibco, Carlsbad, CA, USA) supplemented with $10 \%$ fetal bovine serum (FBS) and $1 \%$ penicillin/streptomycin. Docetaxel-resistant clones, PC3-DR and RV1-DR, were selected by culturing cells with docetaxel in a dose-escalation manner at a 48-h exposure. The initial culture was at a concentration of $2.5 \mathrm{nM}$ docetaxel for PC3 and $1 \mathrm{nM}$ for Rv1. After sensitive clones were no longer present, surviving PC3 and Rv1 cells repopulated the flask and the concentration of docetaxel was increased to 5, 10 and $20 \mathrm{nM}$ for PC3, and 2.5, 5 and $10 \mathrm{nM}$ for Rv1. The process of acquired drug resistance took 4 months, and the docetaxelresistant cell sublines were named PC3-DR and Rv1-DR. In parallel, parental PC3 and Rv1 cells were exposed to dimethyl sulfoxide (DMSO) (vehicle solution) in the same dose-escalation manner. For incubation experiments, docetaxel and calpeptin were purchased from Selleckchem (Houston, TX, USA) and importazole was purchased from Merck Millipore (Billerica, MA, USA).

Western blot analysis. Western blot analysis was carried out as previously described $(25,26)$. Briefly, cell proteins were isolated using RIPA buffer (Beyotime Institute of Biotechnology, Jiangsu, China) supplemented with a protease inhibitor mixture stock solution (Roche Molecular Biochemicals, Mannheim, Germany) and phenylmethanesulfonyl fluoride (PMSF). Equivalent amounts of protein were resolved by $10 \%$ SDS polyacrylamide gels and transferred to polyvinylidene fluoride (PVDF) membranes, which then, were blocked with $5 \%(\mathrm{w} / \mathrm{v})$ nonfat dried-milk and incubated with the indicated primary antibody in Tris-buffered saline overnight at $4^{\circ} \mathrm{C}$. Primary antibodies were FAK antibody (Epitomics, Burlingame, CA, USA), CAPNS1 antibody (ABclonal, Cambridge, MA, USA), AR-V7 monoclonal antibody (A\&G Pharmaceutical, Columbia, MD, USA) (AR-V7 monoclonal antibody recognizes AR-V7, but does not cross-react with intact AR or other AR-Vs), AR antibody (N-20) (Santa Cruz Biotechnology, Santa Cruz, CA, USA) [AR antibody (N-20) recognizes AR-FL, AR-V7 and other non-specific AR-Vs], PSA antibody (Ruiying Biology, Jiangsu, China), lamin B1 antibody, $\alpha$-tubulin antibody (both from Abcam, Cambridge, UK) and $\beta$-actin (Santa Cruz Biotechnology). The blots then were stained with the appropriate horseradish peroxidase-conjugated secondary antibodies, goat anti-rabbit (ProSci, Inc., Poway, CA, USA) and goat anti-mouse (Santa Cruz Biotechnology). Bands were visualized with enhanced chemiluminescence (Thermo Fisher Scientific Inc., Waltham, MA, USA) using the ChemiDoc XRS+ system (Bio-Rad, Hercules, CA, USA).

Cell viability and proliferation assay. To verify the effects on cell viability, we used the 3-(4,5-dimethylthiazol-2-yl)-2,5-diphenyltetrazolium bromide (MTT) assay (27). Cells were seeded at a density of $1 \times 10^{4}$ in 96 -well culture dishes and $24 \mathrm{~h}$ later the medium was removed and replaced with new medium alone (control) or with medium containing the drug. After 
A

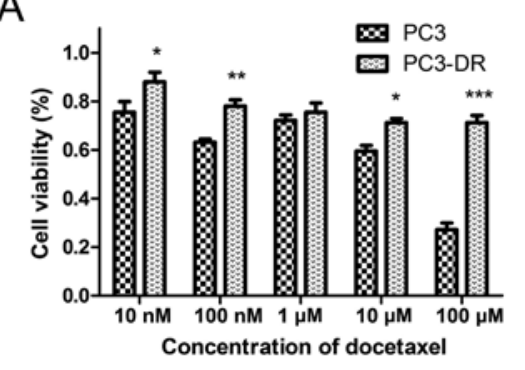

B

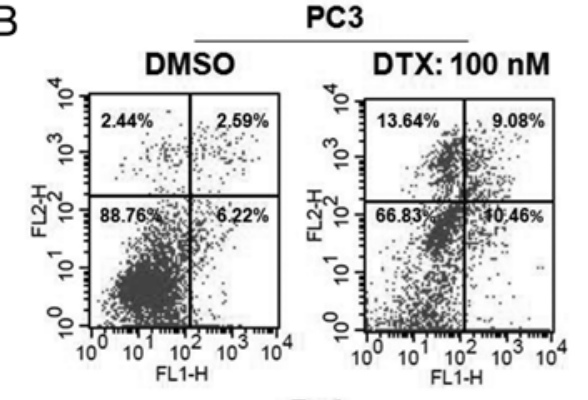

Rv1

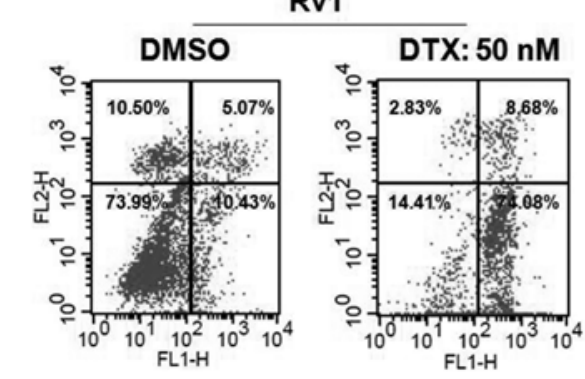

C

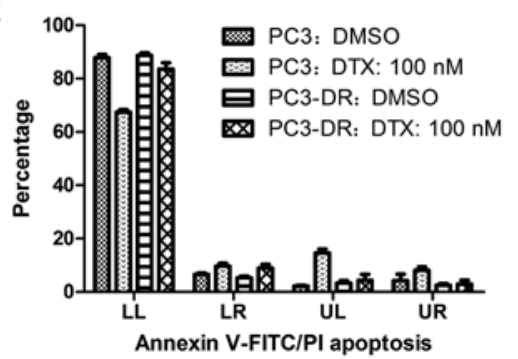

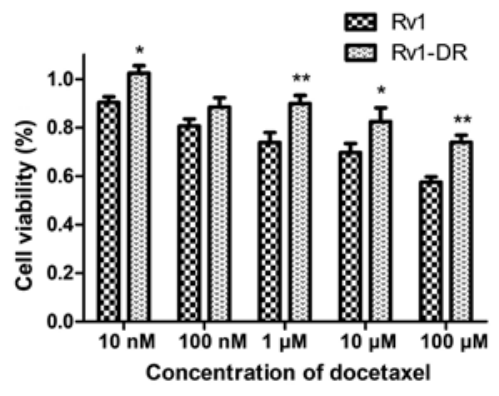

PC3-DR

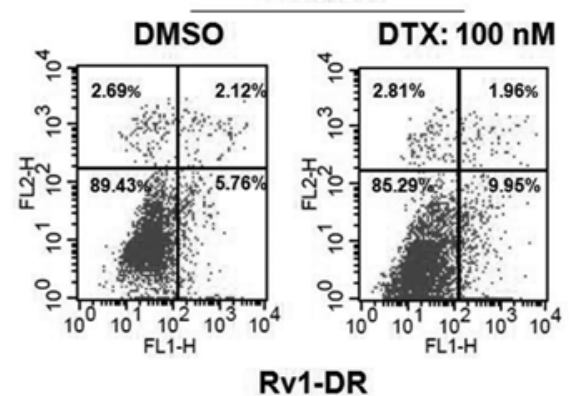

Rv1-DR
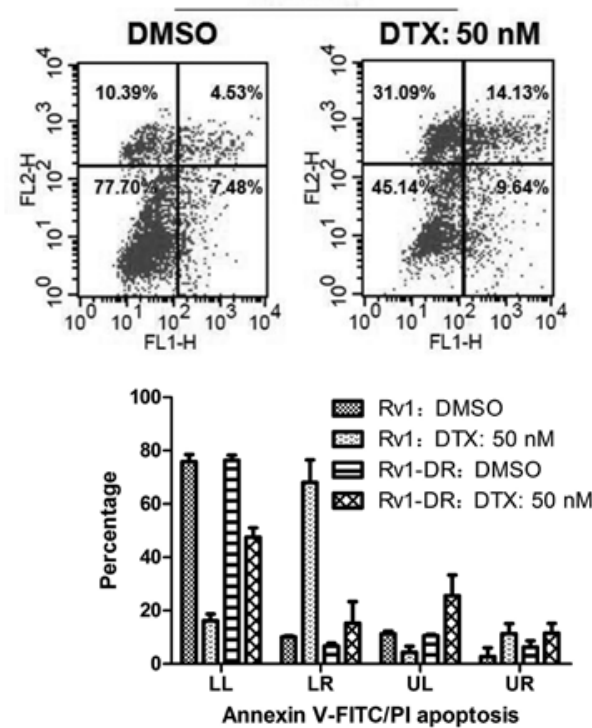

Figure 1. Identification of two in vitro docetaxel-resistant prostate cancer cell sublines. (A) Two docetaxel-resistant prostate cancer cell sublines and their parental cell lines were treated with various concentrations of docetaxel (DTX) for $72 \mathrm{~h}$ and the cell viability was determined using an MTT assay. Data represent the mean $\pm \mathrm{SD}$ values of at least 3 experiments; ${ }^{*} \mathrm{p}<0.05,{ }^{* *} \mathrm{p}<0.01,{ }^{* * *} \mathrm{p}<0.001$. (B) Two docetaxel-resistant prostate cancer cell sublines and their parental cell lines were treated with a certain concentration of docetaxel and cell apoptosis was determined by FACS assay. The lower left quadrant (LL) represents viable cells, the lower right quadrant (LR) represents early apoptotic cells, the upper left quadrant (UL) represents necrotic cells and the upper right quadrant (UR) represents late apoptotic cells. (C) Bar graphs compared the percentage of each quadrant with different treatments. Data represent the mean \pm SD values of at least 3 experiments.

$72 \mathrm{~h}$, the cells were incubated with $0.1 \mu \mathrm{g} / \mu \mathrm{l}$ of aqueous MTT solution for $4 \mathrm{~h}$ at $37^{\circ} \mathrm{C}$. Afterwards, formazan complexes were solubilized by the addition of $150 \mu 1$ of DMSO, and the color absorbance was assessed on a microplate spectrophotometer (Molecular Dynamics) at $450 \mathrm{~nm}$ (test wavelength) and $620 \mathrm{~nm}$ (reference wavelength). The percentage of surviving cells was estimated by dividing the A450-A620 nm of the treated cells by the A450-A620 nm of the control cells. Similarly, cell proliferation was assessed at 24, 48, 72 and $96 \mathrm{~h}$ under different treatments at a density of $3 \times 10^{3}$ cells/well by MTT method as previously described.

Migration assay. The migratory ability of the PC3-DR and Rv1-DR cells under docetaxel, calpeptin, docetaxel combined with calpeptin and DMSO treatment was assessed using a Corning Transwell assay according to the manufacturer's protocol. A total of $1 \times 10^{5}$ cells in $200 \mu$ lof serum-free medium containing drugs were seeded into the upper chamber of the system. The lower chambers were filled with $0.75 \mathrm{ml}$ of $20 \%$ FBS complete medium. After $24 \mathrm{~h}$ of incubation, the cells in the upper chamber were removed with a cotton swab, and the transmigrated cells were fixed in methanol and stained with crystal violet. The stained cells were counted by photographing 5 fields/membrane.

Cell cycle assay. After $24 \mathrm{~h}$ of starvation and synchronization in serum-free medium, the cells were cultured in complete media containing different drugs for 2 days $\left(3 \times 10^{5}\right.$ cells/well), and 
then harvested and washed twice with cold PBS. Subsequently, the cells were fixed in ice-cold $75 \%$ ethanol at $4^{\circ} \mathrm{C}$ overnight and incubated with $50 \mu \mathrm{g} / \mathrm{ml}$ of propidium iodide and $50 \mu \mathrm{g} / \mathrm{ml}$ of RNase A at $37^{\circ} \mathrm{C}$ for $30 \mathrm{~min}$ in the dark. The percentage of cells in the G0/G1, S and G2/M phases was assessed by FACS (BD Biosciences, Franklin Lakes, NJ, USA). Data were analyzed with the ModFit LT software.

Cell apoptosis assay. Cells were cultured in complete media containing different drugs for 2 or 3 days $\left(3 \times 10^{5}\right.$ cells/well). Adherent and detached cells were pooled, washed and labeled with Annexin V-FITC and propidium iodide (PI) using the Annexin V-FITC/PI apoptosis detection kit (KeyGen Biotech, Jiangsu, China) according to the manufacturer's instructions. The percentage of cells displaying Annexin V-FITC/PI staining were measured by FACS (BD Biosciences). Data were analyzed using the ModFit LT software.

Statistical analysis. Statistical comparisons of at least 3 independent measurements were performed using the unpaired Student's t-test with a $95 \%$ confidence interval. For all statistical analyses, results of $\mathrm{p} \leq 0.05$ were considered statistically significant. Data are provided as the mean \pm SD.

\section{Results}

Establishment and identification of 2 in vitro docetaxel-resistant prostate cancer cell sublines. We used CRPC cell lines Rv1 and PC3 as the parental cell lines. The docetaxel-resistant CRPC cell sublines were established by continuous exposure to gradually increasing concentrations of docetaxel. The PC3 and Rv1 docetaxel-resistant cell sublines (named PC3-DR and Rv1-DR) were established with abilities of stable growth and proliferation at a final concentration of 20 and $10 \mathrm{nM}$ docetaxel, respectively. The identification was carried out using MTT assay and a FACS cell apoptosis assay. Compared with their parental cells both docetaxel-resistant cell sublines had a higher survival rate (Fig. 1A), and a lower apoptosis rate (Fig. 1B and C) when exposed to docetaxel.

Activation and overexpression of calpain is induced by docetaxel treatment. To assess the expression of calpain in docetaxel-resistant CRPC cell sublines and parental cell lines, we performed western blot analysis. As shown in Fig. 2A, high expression of calpain S1 was clearly observed in Rv1-DR cells compared with Rv1 cells, but no specific difference in the expression levels of calpain was observed between the PC 3 and PC3-DR cells. Furthermore, to assess the activity of calpain, we chose focal adhesion kinase (FAK) as the marker. FAK (125 kDa) can be specifically cleaved into $90 \mathrm{kDa}$ fragments by calpain (28). As shown in Fig. 2B, the bands at $90 \mathrm{kDa}$ were upregulated under docetaxel treatment $(100 \mathrm{nM})$ in both Rv1 and PC 3 cells; while with calpeptin (a calpain inhibitor) treatment (10 $\mu \mathrm{M}$ for Rv1 and $20 \mu \mathrm{M}$ for PC3 cells), the $90 \mathrm{kDa}$ bands remained steady, and with calpeptin + docetaxel treatment, the $90 \mathrm{kDa}$ bands were 'rescued' from upregulation.

Effects of calpain inhibitor combined with docetaxel on biological functions in PC3-DR and Rvl-DR cells. To assess docetaxel susceptibility in docetaxel-resistant cell sublines we

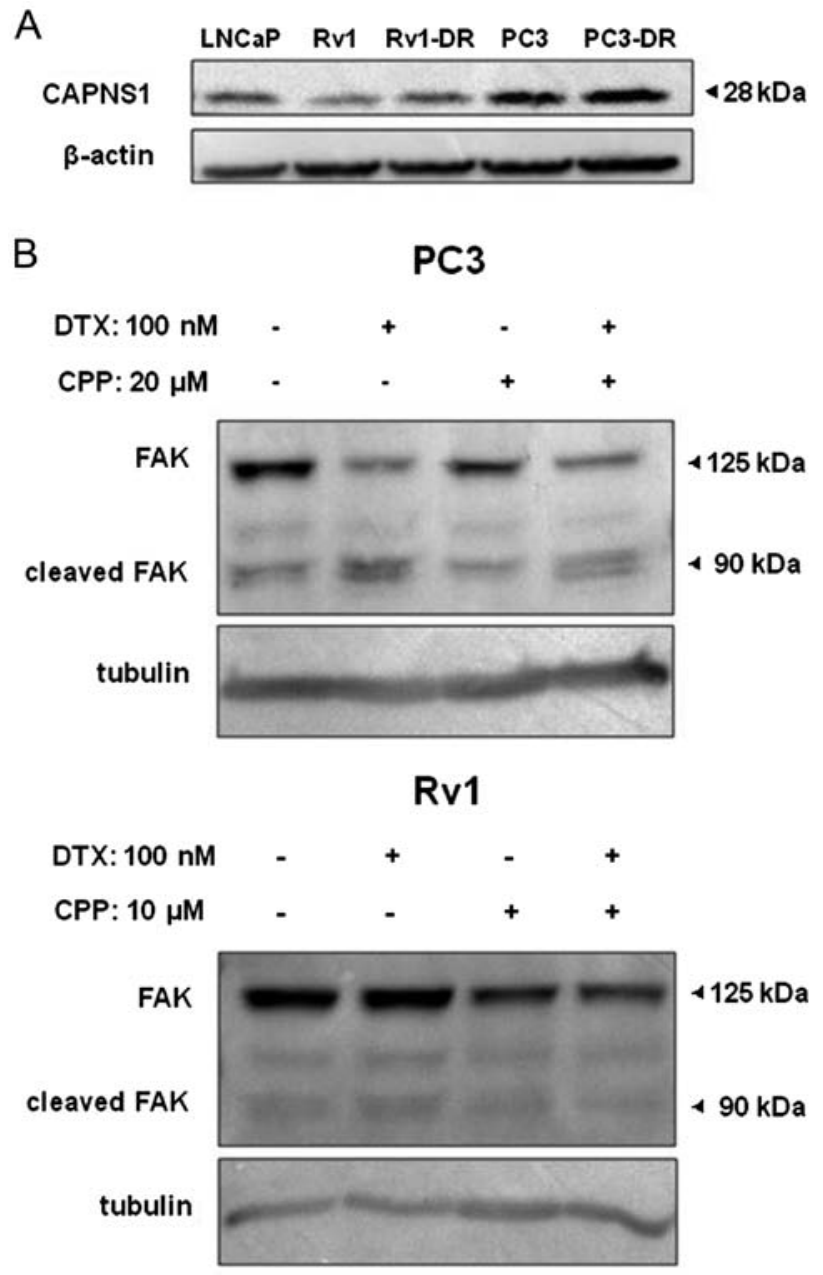

Figure 2. Activation and overexpression of calpain is induced by docetaxel treatment in PC3-DR and Rv1-DR cells. (A) Protein expression of CAPNS1 in Rv1-DR and PC3-DR and their parental cell lines; hormone-naïve prostate cancer (HNPC) cell line (LNCaP) was used as control. (B) Western blotting detection of focal adhesion kinase (FAK) expression and its cleaved fragments with different treatments. DTX represents docetaxel, CPP represents calpeptin (a calpain inhibitor). The experiments were carried out 3 times, obtaining essentially similar results.

used an MTT assay and found that docetaxel susceptibility was significantly increased with exposure to calpeptin $(20 \mu \mathrm{M}$ for PC3-DR and $10 \mu \mathrm{M}$ for Rv1-DR) in both PC3-DR (Fig. 3A) and Rv1-DR (data not shown) cells. To compare the efficiency between calpeptin combined with docetaxel and docetaxel alone in terms of cell proliferation, migration, cell cycle and cell apoptosis, we used MTT and Transwell migration assays, and FACS cell cycle and apoptosis assays, respectively. The results revealed that calpeptin combined with docetaxel had a distinct advantage over docetaxel alone in terms of inhibition of cell proliferation and cell migration in both PC3-DR (Fig. 3B and C) and Rv1-DR (data not shown) cells. G2/M phase arrest was more clearly observed after calpeptin combined with docetaxel treatment for 2 days than docetaxel alone in PC3-DR cells (Fig. 3D), but not in Rv1-DR cells (Fig. 3E). Early stage cell apoptosis was more obvious after calpeptin combined with docetaxel treatment for 2 days than docetaxel alone in Rv1-DR cells (Fig. 3G), but not in PC3-DR cells (Fig. 3F). However, when we prolonged the exposure time to 3 days, early- and late-stage cell apoptosis were both more 

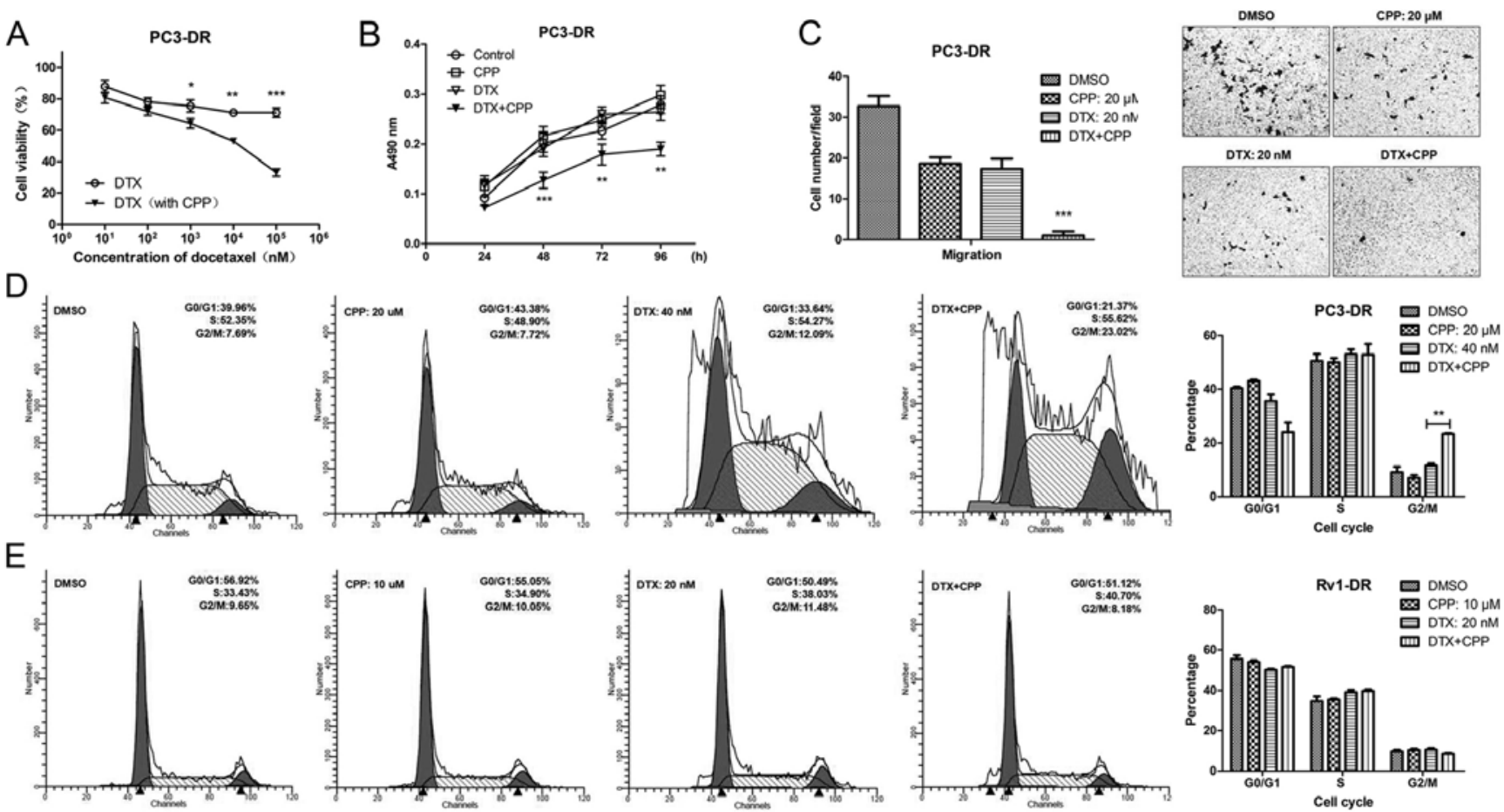

$\mathrm{F}$

PC3-DR (2 days)
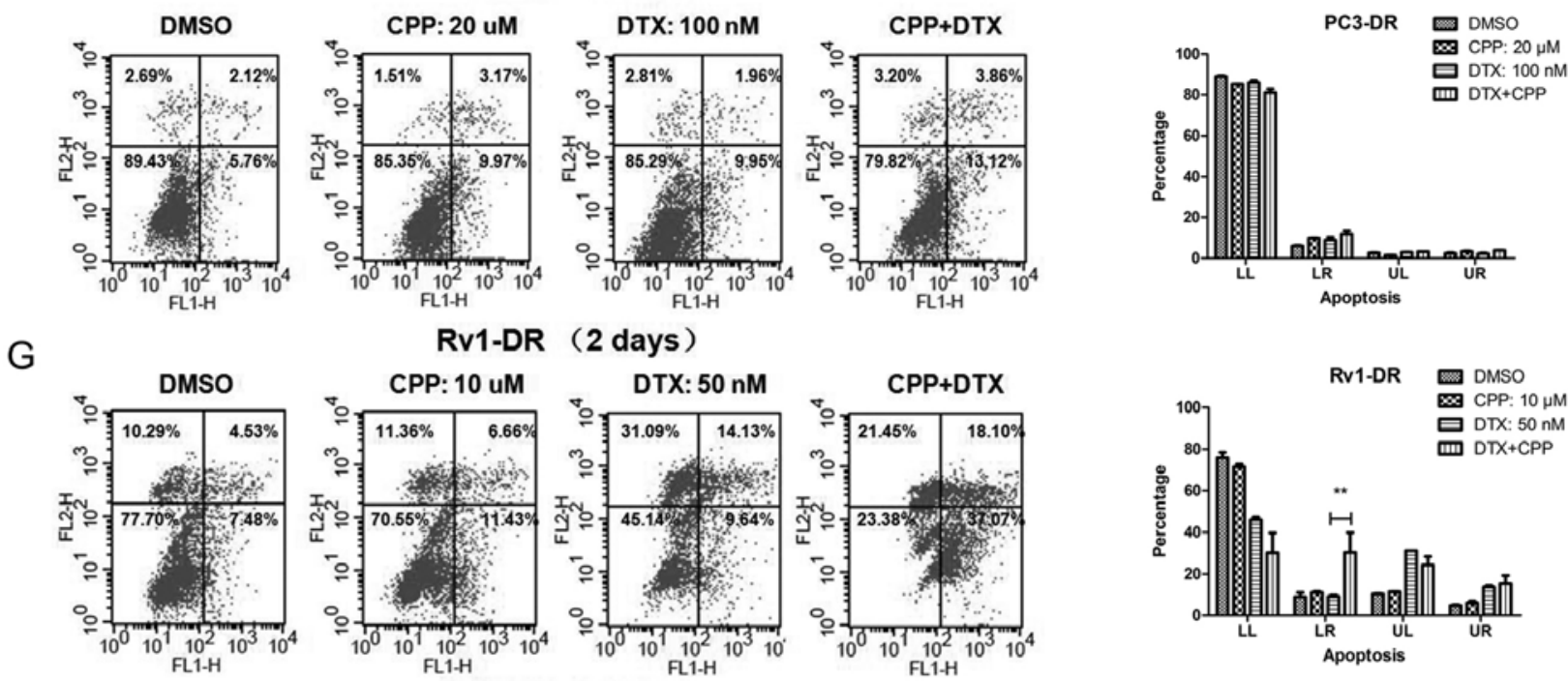

Rv1-DR (2 days)

$\mathrm{H}$
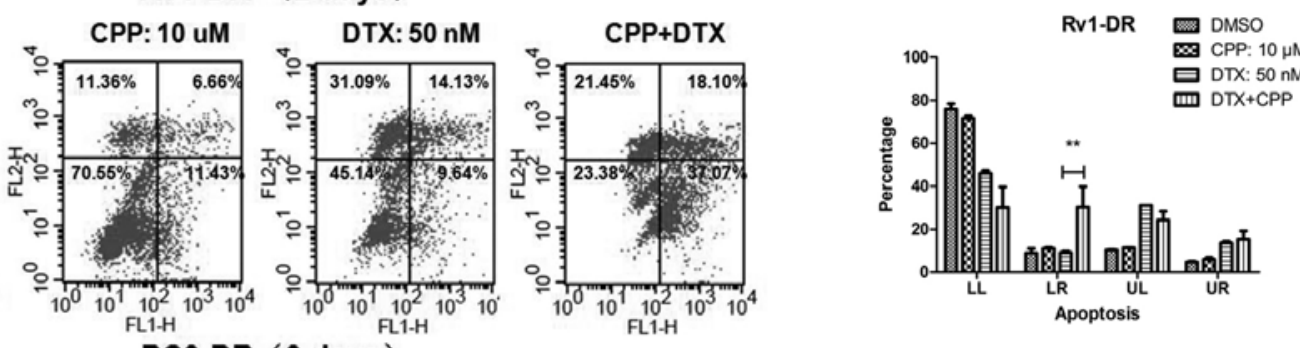

PC3-DR ( 3 days)
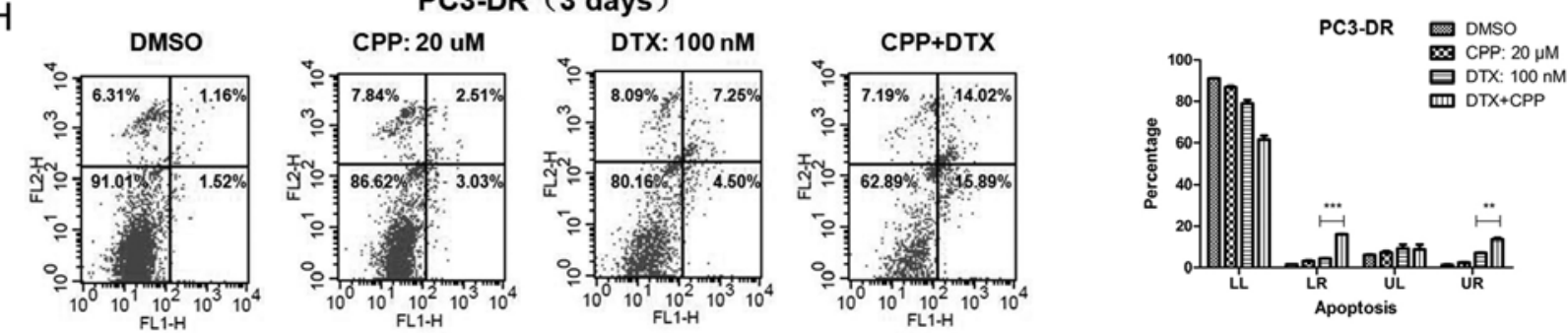

Figure 3. Effects of calpain inhibitor combined with docetaxel on biological functions in PC3-DR and Rv1-DR cells. (A) PC3-DR cells were treated with various concentrations of docetaxel (DTX) for $72 \mathrm{~h}$ with or without CPP pretreatment. The cell viability was determined using an MTT assay. (B) Cell proliferation was monitored after different treatments. Statistical significance was compared between DTX+CPP and DTX alone. (C) Bar graphs comparing the cell migration with different treatments, and the cell images were captured at a magnification of x200. Statistical significance was compared between DTX+CPP and DTX alone. (D and E) Cell cycle of (D) PC3-DR and (E) Rv1-DR were monitored with different treatments. Bar graphs compared the percentage of each cell cycle stage. (F-H) Cell apoptosis was monitored with different treatments. Bar graphs compared the percentage of each quadrant with different treatments. Data represent the mean $\pm \mathrm{SD}$ values of at least 3 experiments; ${ }^{*} \mathrm{p}<0.05,{ }^{* *} \mathrm{p}<0.01,{ }^{* * * *} \mathrm{p}<0.001$.

obvious under calpeptin combined with docetaxel treatment than docetaxel alone in PC3-DR cells (Fig. 3H).
Changes in the expression of AR during docetaxel resistance. To assess the changes in the expression of AR-FL, AR-Vs 

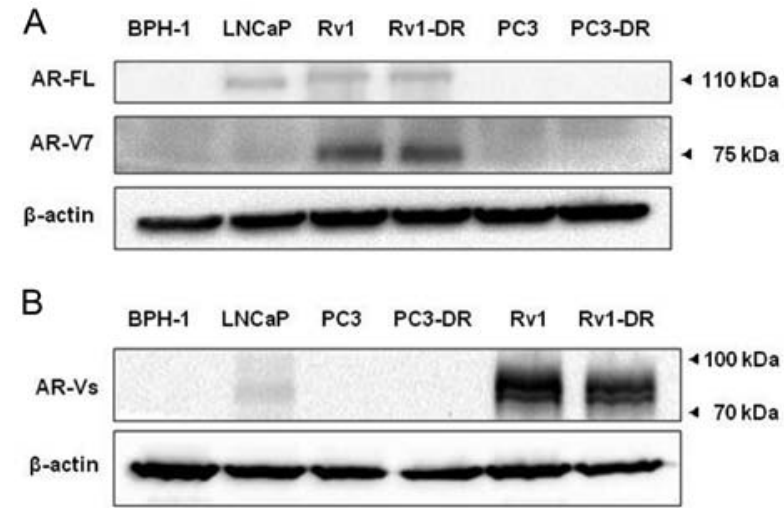

Figure 4. Changes in the expression of androgen receptor (AR) during docetaxel resistance. (A) Western blotting detection of AR-V7 and AR-FL expression in Rv1 and Rv1-DR. BPH-1, LNCaP, PC3 and PC3-DR were used as controls. (B) Western blotting detection of the expression of AR-Vs in Rv1 and Rv1-DR. BPH-1, LNCaP, PC3 and PC3-DR were used as controls. The experiments were carried out 3 times, obtaining essentially similar results.

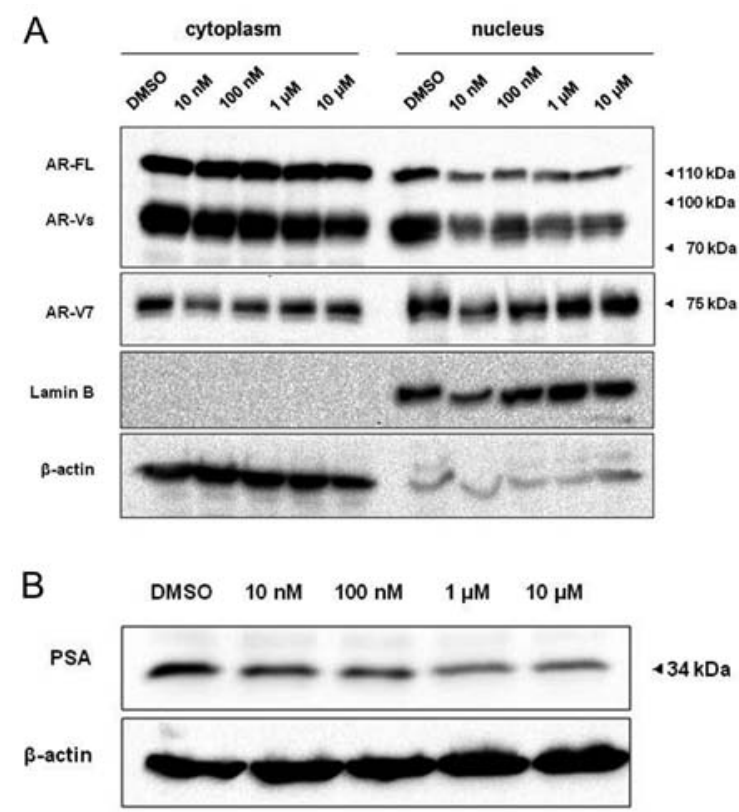

Figure 5. Effects of importin- $\beta$ inhibitor on AR nuclear import and transcriptional activity. (A) Western blotting detection of nuclear and cytoplasmic AR-FL, AR-Vs and AR-V7 expression in Rv1-DR cells after treatment with various concentrations of importazole (a importin- $\beta$ inhibitor) for $48 \mathrm{~h}$. (B) Western blotting detection of PSA expression in Rv1-DR cells after the treatment with various concentrations of importazole for $48 \mathrm{~h}$. The experiments were carried out 3 times, obtaining essentially similar results.

and AR-V7 in Rv1-DR and its parental cell line Rv1, we used prostate cell line BPH-1, LNCaP, PC3 and PC3-DR as controls. Compared with LNCaP and Rv1, we found that the expression of AR-V7 remained at a high level in the Rv1-DR cells (Fig. 4A), while the expression of AR-FL remained very low expression (Fig. 4A), and other non-specific AR-Vs were significantly downregulated (Fig. 4B).

Effects of importin- $\beta$ inhibitor on AR nuclear import and transcriptional activity. To explore the effect of importin- $\beta$ inhibitor on AR nuclear import and transcriptional activity, we used a specific importin- $\beta$ inhibitor (importazole). After a 48-h treatment with gradually increasing concentrations of importazole, nuclear and cytoplasmic protein were isolated using a Nuclear and Cytoplasmic Protein Extraction kit (P0027; Beyotime Institute of Biotechnology) for assessing AR and whole cell protein was isolated for assessing the transcriptional activity (measured with PSA). The results revealed that after using importazole AR-FL and AR-Vs in the nucleus were significantly downregulated at a concentration as low as $10 \mathrm{nM}$ (Fig. 5A) and the expression of PSA decreased slightly with the increase of drug concentration (Fig. 5B); while AR-V7 in the nucleus was not significantly altered. (Fig. 5A).

\section{Discussion}

As docetaxel chemotherapy remains a mainstay in the treatment of CRPC, it is imperative to derive a better understanding of the mechanisms underlying the inherent and acquired docetaxel-resistance, both of which are commonly observed clinically. In the present study we established 2 in vitro docetaxel-resistant prostate cancer cell sublines (PC3-DR and Rv1-DR) by continuously exposing these sublines to gradually increasing concentrations of docetaxel as described in previous studies (29), and identified a docetaxel-resistant character using MTT and FACS cell apoptosis assays.

Previous studies showed that calpain activity played an important role in tumor biology functions. Calpain can promote cell invasion and migration by cleaving the cytoskeleton-related protein FAK (30). However, the role calpain plays in cell survival and apoptosis is still unclear. Calpain can cleave wild-type p53 and induce suppression of p53-dependent apoptosis restrained (31). Calpain is also capable of cleaving anti-apoptotic molecules of the Bcl-2 family leading to the promotion of apoptosis $(32,33)$. This uncertainty may be due to the cellular localization of calpain when it is activated. Recently, it was reported that calpain-dependent proteolysis of the AR into AR-Vs may be androgen-independent mechanism (34-36). Furthermore, calpain can cleave FHL2 and remove the AR and AR-Vs inhibited effect of FHL2, suggesting that AR and AR-Vs are easily imported to the nucleus as transcription factors which drive CRPC progression (37). The promoting role calpain plays in HNPC developing into CRPC has been described, however, the impact of calpain in the process of CRPC developing into docetaxel-resistant CRPC has not been reported. In the present study we assessed the common regulatory subunit CAPNS1 of $\mu$-calpain and m-calpain in 2 docetaxel-resistant CRPC cell sublines and their parental cell lines, and found that CAPNS1 was upregulated in Rv1-DR in comparison to Rv1. In the detection of calpain activity, we chose FAK as the marker, and found that calpain was activated with docetaxel treatment in PC3 and Rv1 cells. Since short-term docetaxel treatment can activate calpain, and long-term docetaxel induction can upregulate calpain expression, can we assume that calpain is a key factor that promotes CRPC resistance to docetaxel? Or is this just an accompanying phenomenon along with other cell physiological functions due to environmental changes? In order to identify this hypothesis, we studied the effects of calpain inhibitor combined with docetaxel on biological functions in PC3-DR and Rv1-DR cells subsequently. We found that calpain inhibition sensitized PC3-DR and Rv1-DR cells to 
docetaxel, and calpeptin combined with docetaxel exhibited a distinct advantage over docetaxel alone in terms of inhibition of cell proliferation, inhibition of cell migration, G2/M phase arrest and induction of cell apoptosis. These results indicate that calpain plays a promoting role in acquired docetaxelresistance of PC3 and Rv1.

Notably, Rv1-DR cells did not exhibit obvious G2/M arrest in calpeptin combined with docetaxel therapy, but exhibited distinct cell apoptosis in combination therapy. These results indicated that Rv1-DR may possess a different mechanism prior to $\mathrm{G} 2 / \mathrm{M}$ arrest inducing cell apoptosis. This mechanism may be the inhibition of the AR-signaling pathway since Rv1-DR cells are very rich in AR $(6,38)$. In addition, we found that PC3-DR cells exhibited an obvious G2/M arrest at a lower concentration of $40 \mathrm{nM}$ of docetaxel and $20 \mu \mathrm{M}$ of calpeptin treatment, but when we increased the concentration of docetaxel to $100 \mathrm{nM}$, we still did not observe distinct cell apoptosis. Subsequently, we prolonged the exposure time from 2 to 3 days, and observed both early- and late-stage cell apoptosis after calpeptin was combined with docetaxel treatment, suggesting that the cell apoptosis induced by the combintion of calpeptin with docetaxel is more time-dependent than concentration-dependent in PC3-DR.

However, it should be noted that, the present study only examined the common regulatory subunit CAPNS1 expression of the heterodimers $\mu$-calpain and m-calpain and further exploration is warranted, whether to expand the molecular mechanism more accurately or to develop highly selective targeting drugs. We used the degradation of only one substrate to determine calpain activity, however further validation of calpain activity with other substrates may be more persuasive. A deeper elucidation of the underlying mechanism in the combination of calpeptin with docetaxel therapy and a in vivo animal experiment evaluating the efficacy and safety of the therapy are both important topics for further study.

To date, a variety of mechanisms involved in docetaxelresistance have been described, but the mechanisms described in literature are mostly common mechanisms which are found in many tumor cells, such as structural and functional changes of microtubules, increasing drug efflux and alterations in apoptosis-related proteins (39), while mechanisms specific to prostate cancer are poorly understood. AR-V7 exists in benign and malignant prostatic tissue, but is mostly enriched in metastatic disease $(21,22)$. The recurrence rate of HNPC after radical surgery has a close and positive correlation to the expression level of AR-V7 in prostatic tissue (21). Moreover, in CRPC bone metastases, overall survival exhibits a strong negative correlation to the expression level of AR-V7 (22). These results indicate that AR-V7 often marks a poor prognosis of malignant prostate cancer. Additionally, previous studies have shown that AR-V7 plays an important role in ADT resistance $(23,40)$, particularly Antonarakis et al demonstrated with strong evidence that the expression of AR-V7 in circulating tumor cells had a negative correlation to abiraterone and enzalutamide treatment outcomes (24). AR-V7 has been widely accepted to be involved in the resistance of ADT, meanwhile, various laboratory studies and clinical studies suggest that there is a cross-resistance phenomenon between docetaxel and second-line ADT drugs (7,9-11), however it is poorly understood whether AR-V7 also confers docetaxel-resistance to prostate cancer cells. Recently, Martin et al observed that a small molecule inhibitor targeting the N-terminal of AR-Vs sensitized prostate cancer cells to docetaxel (41). In addition, Steinestel et al discovered that docetaxel-treated patients had a higher expression of AR-V7 in circulating tumor cells (42). Taking into the consideration the aforementioned facts, we assessed the changes in the expression of AR-FL, AR-Vs and AR-V7 in Rv1-DR and its parental cell line Rv1, and found that the expression of AR-V7 remained at a high level in the docetaxel-resistant cell subline, while full-length the expression of AR remained very low and other non-specific AR-Vs were significantly downregulated. This is a possible consequence of the participation of the constitutively and transcriptionally active AR-V7 in the acquired resistance to docetaxel and overcoming drug toxicity in Rv1 cells.

The human AR is an $110 \mathrm{kDa}, 919$ amino acid protein composed of 4 domains: i) the amino terminal activation domain (NTD); ii) the DNA-binding domain (DBD); iii) the hinge region; and iv) the carboxyl ligand-binding domain (LBD) (43). The NTD includes the majority of the AR and determines androgen specific transcriptional activity (44), while the nucleus localization is a crucial step in the process of the AR signaling pathway (45). AR-V7 lacks the hinge region and LBD, thus making AR-V7 very different from AR-FL (21). Canonical AR nuclear localization signal (NLS) is located in the hinge region that AR-V7 lacks and Chan et al demonstrated that mysterious exon 3-encoded DBD of AR-V7 joined with NLS to reconstitute a bipartite AR NLS which enhanced AR-V7 nuclear localization (46). In prostate cancer cells, AR-FL is transported into the nucleus mediated by microtubules and actin. However, docetaxel which is a microtubule stabilizing agent, can block AR-FL and inhibit the AR signaling pathway by microtubule stabilization. Nevertheless, due to the lack of a hinge structure which binds to microtubules, AR-V7 may have a microtubuleindependent way of translocating to the nucleus, hence AR-V7 nuclear translocation is not regulated by docetaxel $(6,38)$.

Kaku et al assessed the nuclear import abilities of each domain of AR and their mechanisms were related to Ran and importin- $\alpha / \beta$. They discovered that NTD and DBD showed ligand-independent nuclear import ability, and importin- $\beta$ knockdown strongly blocked DBD import (47). Importazole is a small molecule inhibitor of the transport receptor importin- $\beta$ (48). A former study suggested that importazole blocked the nuclear import of AR-V7 as was determined by FRAP assay, however, docetaxel was only capable of blocking nuclear import of AR-FL, but not AR-V7 (49). Moreover, the concentration they used to treat the cells was as much as $50 \mu \mathrm{M}$ and under such a high concentration it is hard to guarantee that the results of the experiment are still caused by the normal pharmacological effects of importazole. Furthermore it is hard to achieve such a concentration in human prostate cells. In the present study we used gradient increasing doses of importazole to treat Rv1-DR cells for $48 \mathrm{~h}$. The nucleus and cytoplasm proteins were isolated to assess the distribution of AR-FL, AR-Vs and AR-V7, in order to study the effect of importazole on their nuclear import. To illustrate that the isolation of nuclear and cytoplasmic protein was successful, we chose $\beta$-actin as a cytoplasmic marker and lamin B as a nuclear marker. We found that gradient increasing doses of importazole could not block the nuclear import of AR-V7. 
Furthermore, we studied the effect of importazole on AR transcriptional activity, and observed that PSA was only slightly downregulated with the increase of drug concentration. Although, importazole could block the nuclear import of AR-FL and non-specific AR-Vs significantly at a concentration as low as $10 \mathrm{nM}$, the AR transcriptional activity was slightly inhibited. These results indicated that the expression of AR-FL which remains at a low level and non-specific AR-Vs which are significantly downregulated are not the key factors of the AR signaling pathway in Rv1-DR. Moreover, AR-V7 which remains highly expressed in Rv1-DR and is not regulated by importazole may be the key factor of the AR signaling pathway and confer docetaxel-resistance to Rv1-DR cells.

We have to point out that only using PSA expression to illustrate the AR transcriptional activity is not convincing enough, we could also use other AR target gene products or luciferase assay to assess AR transcriptional activity. We suggested that importazole can block the nuclear import of AR-FL and AR-Vs. A Co-IP assay study on the interaction between importin- $\beta$ and AR-FL as well as AR-Vs may provide us with more powerful evidence. Additionally, the effect of importazole on the biological functions of prostate cancer has not been reported yet. These issues are all urgent and warrant elucidation in the future.

In the present study we demonstrated that overexpression and activation of calpain confers chemoresistance to CRPC cells. Targeting calpain sensitized PC3-DR and Rv1-DR cells to docetaxel. The calpain-induced insensitivity to docetaxel was partially reversed by calpeptin (a calpain inhibitor). These results concerning the biological functions collectively highlight an important role for calpain in the modulation of docetaxelresistance of prostate cancer cells. Moreover, we suggested that AR-V7 may play a promoting role in CRPC-acquired docetaxelresistance. An importin- $\beta$ inhibitor was only capable of slightly decreasing the transcriptional activity of the AR signaling pathway via blocking nuclear import of AR-FL and some nonspecific AR-Vs, instead of AR-V7.

In conclusion, we provided evidence that calpain and AR-V7 play crucial roles in mediating the chemoresistance of CRPC cells to docetaxel. In addition, the expression status of calpain and AR-V7 could potentially be used as biomarkers to aid treatment selection and sequencing in prostate cancer. Furthermore, targeting calpain and AR-V7 may thus have potential benefits in improving the effectiveness of chemotherapy and overcoming docetaxel-resistance in prostate cancer.

\section{Acknowledgements}

This research was supported by the National Natural Science Foundation of China (Grant no. 30872924, 81072095 and 81372760), Hubei Province Scientific and Technical International Joint Project (Grant no. 2015BHE007) to Professor Xiaoping Zhang. The authors also thank Professor Shaoyong Chen (Beth Israel Deaconess Medical Center and Harvard Medical School, Boston, USA) for providing the Rv1 cell line.

\section{References}

1. Harris WP, Mostaghel EA, Nelson PS and Montgomery B Androgen deprivation therapy: Progress in understanding mechanisms of resistance and optimizing androgen depletion. Nat Clin Pract Urol 6: 76-85, 2009.
2. Tannock IF, de Wit R, Berry WR, Horti J, Pluzanska A, Chi KN Oudard S, Théodore C, James ND, Turesson I, et al; TAX 327 Investigators: Docetaxel plus prednisone or mitoxantrone plus prednisone for advanced prostate cancer. N Engl J Med 351: 1502-1512, 2004.

3. Loriot Y and Fizazi K: Taxanes: Still a major weapon in the armamentarium against prostate cancer. Eur Urol 63: 983-985, 2013.

4. Heidenreich A, Bastian PJ, Bellmunt J, Bolla M, Joniau S, van der Kwast T, Mason M, Matveev V, Wiegel T, Zattoni F, et al; European Association of Urology: EAU guidelines on prostate cancer. Part II: Treatment of advanced, relapsing, and castrationresistant prostate cancer. Eur Urol 65: 467-479, 2014.

5. Jordan MA and Wilson L: Microtubules as a target for anticancer drugs. Nat Rev Cancer 4: 253-265, 2004.

6. Zhu ML, Horbinski CM, Garzotto M, Qian DZ, Beer TM and Kyprianou N: Tubulin-targeting chemotherapy impairs androgen receptor activity in prostate cancer. Cancer Res 70: 7992-8002, 2010.

7. Mezynski J, Pezaro C, Bianchini D, Zivi A, Sandhu S, Thompson E, Hunt J, Sheridan E, Baikady B, Sarvadikar A, et al: Antitumour activity of docetaxel following treatment with the CYP17A1 inhibitor abiraterone: Clinical evidence for cross-resistance? Ann Oncol 23: 2943-2947, 2012.

8. Schweizer MT, Zhou XC, Wang H, Bassi S, Carducci MA, Eisenberger MA and Antonarakis ES: The influence of prior abiraterone treatment on the clinical activity of docetaxel in men with metastatic castration-resistant prostate cancer. Eur Urol 66: 646-652, 2014

9. van Soest RJ, van Royen ME, de Morrée ES, Moll JM, Teubel W, Wiemer EA, Mathijssen RH, de Wit R and van Weerden WM: Cross-resistance between taxanes and new hormonal agents abiraterone and enzalutamide may affect drug sequence choices in metastatic castration-resistant prostate cancer. Eur $\mathbf{J}$ Cancer 49: 3821-3830, 2013.

10. Nadal R, Zhang Z, Rahman H, Schweizer MT, Denmeade SR, Paller CJ, Carducci MA, Eisenberger MA and Antonarakis ES: Clinical activity of enzalutamide in docetaxel-naïve and docetaxel-pretreated patients with metastatic castration-resistant prostate cancer. Prostate 74: 1560-1568, 2014.

11. Cheng HH, Gulati R, Azad A, Nadal R, Twardowski P, Vaishampayan UN, Agarwal N, Heath EI, Pal SK, Rehman HT, et al: Activity of enzalutamide in men with metastatic castration-resistant prostate cancer is affected by prior treatment with abiraterone and/ or docetaxel. Prostate Cancer Prostatic Dis 18: 122-127, 2015.

12. Goll DE, Thompson VF, Li H, Wei W and Cong J: The calpain system. Physiol Rev 83: 731-801, 2003.

13. Kimura Y, Koga H, Araki N, Mugita N, Fujita N, Takeshima H, Nishi T, Yamashima T, Saido TC, Yamasaki T, et al: The involvement of calpain-dependent proteolysis of the tumor suppressor NF2 (merlin) in schwannomas and meningiomas. Nat Med 4: 915-922, 1998.

14. Braun C, Engel M, Seifert M, Theisinger B, Seitz G, Zang KD and Welter C: Expression of calpain I messenger RNA in human renal cell carcinoma: Correlation with lymph node metastasis and histological type. Int J Cancer 84: 6-9, 1999.

15. Lakshmikuttyamma A, Selvakumar P, Kanthan R, Kanthan SC and Sharma RK: Overexpression of $m$-calpain in human colorectal adenocarcinomas. Cancer Epidemiol Biomarkers Prev 13: 1604-1609, 2004.

16. Mamoune A, Luo JH, Lauffenburger DA and Wells A: Calpain-2 as a target for limiting prostate cancer invasion. Cancer Res 63: 4632-4640, 2003.

17. Rios-Doria J, Day KC, Kuefer R, Rashid MG, Chinnaiyan AM, Rubin MA and Day ML: The role of calpain in the proteolytic cleavage of E-cadherin in prostate and mammary epithelial cells. J Biol Chem 278: 1372-1379, 2003.

18. Liu T, Mendes DE and Berkman CE: Prolonged androgen deprivation leads to overexpression of calpain 2: Implications for prostate cancer progression. Int J Oncol 44: 467-472, 2014.

19. Jorfi S, Ansa-Addo EA, Kholia S, Stratton D, Valley S, Lange S and Inal J: Sci Rep 5: 13006, 2015.

20. Dehm SM, Schmidt LJ, Heemers HV, Vessella RL and Tindall DJ: Splicing of a novel androgen receptor exon generates a constitutively active androgen receptor that mediates prostate cancer therapy resistance. Cancer Res 68: 5469-5477, 2008.

21. Guo Z, Yang X, Sun F, Jiang R, Linn DE, Chen H, Chen H, Kong X, Melamed J, Tepper CG, et al: A novel androgen receptor splice variant is up-regulated during prostate cancer progression and promotes androgen depletion-resistant growth. Cancer Res 69: 2305-2313, 2009. 
22. Hörnberg E, Ylitalo EB, Crnalic S, Antti H, Stattin P, Widmark A, Bergh A and Wikström P: Expression of androgen receptor splice variants in prostate cancer bone metastases is associated with castration-resistance and short survival. PLoS One 6: e19059, 2011.

23. Li Y, Chan SC, Brand LJ, Hwang TH, Silverstein KA and Dehm SM: Androgen receptor splice variants mediate enzalutamide resistance in castration-resistant prostate cancer cell lines. Cancer Res 73: 483-489, 2013.

24. Antonarakis ES, Lu C, Wang H, Luber B, Nakazawa M, Roeser JC, Chen Y, Mohammad TA, Chen Y, Fedor HL, et al: AR-V7 and resistance to enzalutamide and abiraterone in prostate cancer. N Engl J Med 371: 1028-1038, 2014.

25. Zhang X, Jin TG, Yang H, DeWolf WC, Khosravi-Far R and Olumi AF: Persistent c-FLIP(L) expression is necessary and sufficient to maintain resistance to tumor necrosis factor-related apoptosis-inducing ligand-mediated apoptosis in prostate cancer. Cancer Res 64: 7086-7091, 2004.

26. Xu S, Tao Z, Hai B, Liang H, Shi Y, Wang T, Song W, Chen Y, OuYang J, Chen J, et al: miR-424(322) reverses chemoresistance via T-cell immune response activation by blocking the PD-L1 immune checkpoint. Nat Commun 7: 11406, 2016.

27. Xu S, Wang T, Song W, Jiang T, Zhang F, Yin Y, Jiang SW, Wu K, Yu Z, Wang C, et al: The inhibitory effects of AR/miR-190a/ YB-1 negative feedback loop on prostate cancer and underlying mechanism. Sci Rep 5: 13528, 2015.

28. Cooray P, Yuan Y, Schoenwaelder SM, Mitchell CA, Salem HH and Jackson SP: Focal adhesion kinase $\left(\mathrm{pp} 125^{\mathrm{FAK}}\right)$ cleavage and regulation by calpain. Biochem J 318: 41-47, 1996.

29. Domingo-Domenech J, Vidal SJ, Rodriguez-Bravo V, CastilloMartin M, Quinn SA, Rodriguez-Barrueco R, Bonal DM, Charytonowicz E, Gladoun N, de la Iglesia-Vicente J, et al: Suppression of acquired docetaxel resistance in prostate cancer through depletion of Notch- and Hedgehog-dependent tumorinitiating cells. Cancer Cell 22: 373-388, 2012.

30. Chan KT, Bennin DA and Huttenlocher A: Regulation of adhesion dynamics by calpain-mediated proteolysis of focal adhesion kinase (FAK). J Biol Chem 285: 11418-11426, 2010.

31. Kubbutat MH and Vousden KH: Proteolytic cleavage of human p53 by calpain: A potential regulator of protein stability. Mol Cell Biol 17: 460-468, 1997.

32. Wood DE, Thomas A, Devi LA, Berman Y, Beavis RC, Reed JC and Newcomb EW: Bax cleavage is mediated by calpain during drug-induced apoptosis. Oncogene 17: 1069-1078, 1998.

33. Gao G and Dou QP: N-terminal cleavage of bax by calpain generates a potent proapoptotic $18-\mathrm{kDa}$ fragment that promotes bcl-2-independent cytochrome $c$ release and apoptotic cell death. J Cell Biochem 80: 53-72, 2000.

34. Chen H, Libertini SJ, Wang Y, Kung HJ, Ghosh P and Mudryj M: ERK regulates calpain 2-induced androgen receptor proteolysis in CWR22 relapsed prostate tumor cell lines. J Biol Chem 285: 2368-2374, 2010.

35. Pelley RP, Chinnakannu K, Murthy S, Strickland FM, Menon M, Dou QP, Barrack ER and Reddy GP: Calmodulin-androgen receptor (AR) interaction: Calcium-dependent, calpain-mediated breakdown of $\mathrm{AR}$ in $\mathrm{LNCaP}$ prostate cancer cells. Cancer Res 66: 11754-11762, 2006
36. Libertini SJ, Tepper CG, Rodriguez V, Asmuth DM, Kung HJ and Mudryj M: Evidence for calpain-mediated androgen receptor cleavage as a mechanism for androgen independence. Cancer Res 67: 9001-9005, 2007.

37. McGrath MJ, Binge LC, Sriratana A, Wang H, Robinson PA, Pook D, Fedele CG, Brown S, Dyson JM, Cottle DL, et al: Regulation of the transcriptional coactivator FHL2 licenses activation of the androgen receptor in castrate-resistant prostate cancer. Cancer Res 73: 5066-5079, 2013.

38. Darshan MS, Loftus MS, Thadani-Mulero M, Levy BP, Escuin D, Zhou XK, Gjyrezi A, Chanel-Vos C, Shen R, Tagawa ST, et al: Taxane-induced blockade to nuclear accumulation of the androgen receptor predicts clinical responses in metastatic prostate cancer. Cancer Res 71: 6019-6029, 2011.

39. Antonarakis ES and Armstrong AJ: Evolving standards in the treatment of docetaxel-refractory castration-resistant prostate cancer. Prostate Cancer Prostatic Dis 14: 192-205, 2011.

40. Mostaghel EA, Marck BT, Plymate SR, Vessella RL, Balk S, Matsumoto AM, Nelson PS and Montgomery RB: Resistance to CYP17A1 inhibition with abiraterone in castration-resistant prostate cancer: Induction of steroidogenesis and androgen receptor splice variants. Clin Cancer Res 17: 5913-5925, 2011.

41. Martin SK, Banuelos CA, Sadar MD and Kyprianou N: $\mathrm{N}$-terminal targeting of androgen receptor variant enhances response of castration resistant prostate cancer to taxane chemotherapy. Mol Oncol 9: 628-639, 2014.

42. Steinestel J, Luedeke M, Arndt A, Schnoeller TJ, Lennerz JK, Wurm C, Maier C, Cronauer MV, Steinestel K and Schrader AJ: Detecting predictive androgen receptor modifications in circulating prostate cancer cells. Oncotarget: Apr 23, 2015 (Epub ahead of print).

43. Yong EL, Ghadessy F, Wang Q, Mifsud A and Ng SC: Androgen receptor transactivation domain and control of spermatogenesis. Rev Reprod 3: 141-144, 1998.

44. Jenster G, van der Korput HA, Trapman J and Brinkmann AO: Identification of two transcription activation units in the $\mathrm{N}$-terminal domain of the human androgen receptor. J Biol Chem 270: 7341-7346, 1995.

45. Gelmann EP: Molecular biology of the androgen receptor. J Clin Oncol 20: 3001-3015, 2002.

46. Chan SC, Li Y and Dehm SM: Androgen receptor splice variants activate androgen receptor target genes and support aberrant prostate cancer cell growth independent of canonical androgen receptor nuclear localization signal. J Biol Chem 287: 19736-19749, 2012.

47. Kaku N,Matsuda K, Tsujimura A and Kawata M: Characterization of nuclear import of the domain-specific androgen receptor in association with the importin alpha/beta and Ran-guanosine 5'-triphosphate systems. Endocrinology 149: 3960-3969, 2008.

48. Soderholm JF, Bird SL, Kalab P, Sampathkumar Y, Hasegawa K, Uehara-Bingen $\mathrm{M}$, Weis $\mathrm{K}$ and Heald R: Importazole, a small molecule inhibitor of the transport receptor importin- $\beta$. ACS Chem Biol 6: 700-708, 2011.

49. Zhang G, Liu X, Li J, Ledet E, Alvarez X, Qi Y, Fu X, Sartor O, Dong Y and Zhang $\mathrm{H}$ : Androgen receptor splice variants circumvent AR blockade by microtubule-targeting agents. Oncotarget 6: 23358-23371, 2015. 